\title{
Effect of Stereoscopic Comprehensive Therapy of Zhuang Medicine on Inflammatory Factor IL-6 in Patients with Rheumatoid Arthritis
}

\author{
Xiusong Tang ${ }^{1}$, Yanqiang Chen ${ }^{2}$, Yuzhi Shang1, Yulei Fu ${ }^{1}$, Yuzhou Pang ${ }^{1 *}$ \\ ${ }^{1}$ Guangxi University of Traditional Chinese Medicine, Nanning 530200, Guangxi Province, China \\ ${ }^{2}$ The First Affiliated Hospital of Guangxi University of Chinese Medicine, Nanning 530023, Guangxi Province, China
}

Funding: 1. National Key Research and Development Program of China (No. : 2017YFC1704004); 2. Guangxi First-class Discipline Construction Open Project of Guangxi University of Chinese Medicine (No.: 2018XK065).

\begin{abstract}
[Abstract] Objective: To study the effect of Stereoscopic Comprehensive of Therapy Zhuang Medicine on IL-6 in serum of patients with rheumatoid arthritis. Methods: Sixty rheumatoid arthritis patients who met the inclusion criteria were selected and randomly divided into a control group and a treatment group using a random number table. Among them, 30 cases in the control group were treated with Western medicine, and 30 cases in the treatment group were treated with Western medical and Stereoscopic Comprehensive Therapy of Zhuang Medicine. The observation period was 8 weeks. Results: After 8 weeks of treatment, the level of IL-6 in the treatment group and the control group decreased, and the treatment group was better than the control group $(P<0.05)$. Conclusion: The Stereoscopic Comprehensive of Zhuang Medicine can reduce the level of IL-6 in the serum of patients with rheumatoid arthritis, and has a good regulating effect on the inflammation of rheumatoid arthritis.
\end{abstract}

Key words: Rheumatoid arthritis; Stereoscopic comprehensive therapy of Zhuang medicine; Inflammatory factors; Interleukin 6

Publication date: May, 2021; Publication online: 31 May, 2021

*Corresponding author: Yuzhou Pang, pangyz422@sina.com

Rheumatoid Arthritis (RA) is a universal, systemic, and inflammatory disease, characterized by persistent synovitis and progressive joint destruction, swelling, pain, stiffness, and limited range of motion ${ }^{[1,2]}$. RA affects about $1 \%$ of the population worldwide ${ }^{[3]}$. The pathological mechanism of RA is not yet clear, and it is believed to be related to genetic susceptibility, environmental factors and changes in immune function $^{[4]}$. Clinical treatment of RA mainly focuses on relieving joint pain and improving quality of life, and delaying the destruction of bone and joint is the main goal. Commonly used drugs include non-steroidal anti-inflammatory drugs, glucocorticoids and anti-rheumatic drugs, etc ${ }^{[5]}$. Interleukin 6 (IL-6), as a pleiotropic cytokine, plays an important role in pre-adaptive and adaptive immune responses in its specific immunomodulatory function ${ }^{[6]}$. Studies have found that IL-6 plays an important role in RA, especially in chronic inflammation. IL-6 mainly destroys the balance of osteoblasts and osteoclasts, leading to osteoporosis in patients and aggravating the progression of $\mathrm{RA}^{[7]}$. The discovery of IL-6 inhibitors, such as tozumab, has brought great changes to the clinical treatment of RA, and its effectiveness has been widely recognized. Therefore, as one of the biomarkers of RA, the study of IL-6 has important clinical significance.

Zhuang medicine is one of the traditional Chinese medicine. Zhuang medicine believes that RA belongs to the category of "flourishing" and that "network prevents the cause of $\mathrm{Bi}^{\prime}$ is one of the main factors ${ }^{[8]}$. The treatment of 
RA by Zhuang medicine includes bamboo pot therapy, needle picking therapy, medicine line point moxibustion therapy, internal administration of Zhuang medicine and comprehensive treatment, and has achieved good therapeutic effect. The three-dimensional comprehensive therapy of zhuang medicine is a kind of therapy which is summarized and extracted from the clinical practice experience of professor pang yuzhou, the academic leader of zhuang medicine. The therapy mainly consists of "internal administration of zhuang medicine longzhuo tongbi prescription, external application of zhuang medicine thermal probe point acupuncture, and coordination of zhuang medicine food therapy prescription". The purpose of this study is to observe the expression level of IL-6 in serum of patients with rheumatoid arthritis, so as to preliminarily evaluate and discuss the effectiveness and mechanism of three-dimensional comprehensive therapy of Zhuang Medicine in the treatment of rheumatoid arthritis, in order to provide reference for the clinical prevention and treatment of rheumatoid arthritis.

\section{Materials and methods}

\subsection{Source of cases}

The study in accordance with the requirements of the medical ethics and hospital ethics committee approval, on the basis of the cases included in the main from March 2019 to December 2019 in the first affiliated hospital of Guangxi medical university during the period of rheumatism of inpatient and outpatient clinic patients, in strict accordance with the standards for these patients in the study of screening, finally into the study of 60 patients with RA were involved in this experiment.

\subsection{Case diagnosis and discharge criteria}

\subsubsection{Case diagnosis criteria}

The case diagnostic criteria in this study mainly included the diagnostic criteria of Zhuang medicine and Western medicine, among which the diagnostic criteria of Zhuang medicine were formulated according to the Guidelines for the Diagnosis and Treatment of Diseases and Synonyms of Chinese Zhuang Medicine, which was edited by Zhong Ming and published in 2009. Western diagnostic criteria were based on the classification criteria of rheumatoid arthritis jointly published by the American College of Rheumatology (ACR)/ European League Against Rheumatology (EULAR) in $2009^{[9]}$.

\subsubsection{Case inclusion criteria}

(1) Those who meet the case diagnosis criteria set out in 1.2.1; (2) Age ranges from 18 to 65; (3) Those who voluntarily participate in the study and sign the informed consent.

\subsubsection{Case exclusion criteria}

(1) Patients with other autoimmune diseases, such as systemic lupus erythematosus, severe knee osteoarthritis and other rheumatic diseases; (2) Patients with severe joint deformity; (3) Patients with multiple system organ dysfunction and other serious diseases; (4) People with history of allergy to the drugs used in this experiment and acupuncture sickness; (5) Pregnant or lactating women; (6) People who are not taking the drug for the first time or are taking other anti-rheumatic drugs; (7) Patients deemed by clinicians to be unsuitable for participation in this clinical trial.

\subsubsection{Elimination and shedding criteria}

Those who do not meet the inclusion criteria after inclusion shall be excluded;In the process of clinical trial, poor compliance, automatic withdrawal, serious adverse reactions should not be continued subjects.

\subsection{Treatment methods}

Methotrexate tablets and leflunomide tablets were used as basic antirheumatic drugs in both groups. Methotrexate tablet, orally, once a week, 10mg each time; Leflunomide Tablets, 10mg once daily, orally. The control group was given basic Western medicine treatment, and the treatment group was given Zhuang medicine Longzhuotongbi granules orally $(15 \mathrm{~g} /$ package, twice a day), combined with Zhuang medicine. Thermin Tan acupoint acupuncture therapy (twice a week), and Zhuang medicine diet therapy package (80g/ package, once a week) for recuperation on the basis of Western medicine treatment. The observation period was 8 weeks.

\subsection{Observation indexes}

At the end of the 8 th week of treatment, $5 \mathrm{ml}$ of fasting blood samples were extracted from each group using procoagulant tubes and centrifuged at $3000 \mathrm{r} / \mathrm{min}$ at $4{ }^{\circ} \mathrm{C}$ for $5 \mathrm{~min}$. Serum was collected and stored at $-80{ }^{\circ} \mathrm{C}$ for later use. Enzyme-linked immunoassay was used to detect the expression of inflammatory cytokine IL-6 in serum of patients in each group. The kit was enzyme-linked immunoassay (Shanghai Enzyme-linked Biology), and the operation was strictly in accordance with the reagent instructions $^{[10]}$.

\subsection{Statistical methods}


SPSS 25.0 was used for data statistical analysis. Measurement data were expressed as mean \pm collimation $(x \pm s)$, and $t$ test was used for comparison between groups. Enumeration data were expressed as rate $(\%)$, and $\chi^{2}$ test was used for comparison between groups. $P<0.05$ was considered to be statistically significant.

\section{Results}

There was no significant difference in general data between the control group and the treatment group (P\&GT; 0.05), as shown in Table 1. Before treatment, there was no significant difference in serum IL- 6 content between the two groups (P\&GT; 0.05);After treatment, the serum IL-6 content in the treatment group was significantly decreased (P\&LT; 0.01), serum IL-6 content in control group was significantly decreased (P\&LT; 0.05), and the reduction in the treatment group was significantly better than that in the control group, the difference between the two groups was statistically significant (P\&LT; 0.05). Are shown in Table 2.

Table 1. Comparison of general data of patients with rheumatoid arthritis between the two groups

\begin{tabular}{|c|c|c|c|c|c|c|c|c|}
\hline \multirow{2}{*}{ Group } & \multirow{2}{*}{$\mathrm{N}$} & \multirow{2}{*}{$\begin{array}{c}\text { Gender } \\
\text { Male/Female }\end{array}$} & \multicolumn{3}{|c|}{ Age ( Year ) } & \multicolumn{3}{|c|}{ The course of the disease/Month } \\
\hline & & & Biggest & Minimum & Average $(\mathrm{x} \pm \mathrm{s})$ & Shortest $\left(\mathrm{x}^{-} \pm \mathrm{s}\right)$ & Longest & Average \\
\hline The control group & 30 & $10 / 20$ & 58 & 27 & $44 \pm 15.56$ & 12 & 157 & $81.00 \pm 11.75$ \\
\hline The treatment group & 30 & $12 / 18$ & 59 & 25 & $43 \pm 15.71$ & 9 & 160 & $79.00 \pm 16.51$ \\
\hline
\end{tabular}

Table 2. Comparison of IL-6 between the two groups before and after treatment $\quad(\mathrm{x} \pm \mathrm{s})$

\begin{tabular}{ccccc}
\hline Group & $\mathrm{N}$ & \multicolumn{3}{c}{ IL-6(pg/ml) } \\
\cline { 3 - 5 } & & Before treatment & After treatment & \\
\hline The control group & 30 & $456.78 \pm 33.27$ & $364.54 \pm 43.69$ & 0.026 \\
The treatment group & 30 & $461.51 \pm 31.76$ & $279.65 \pm 33.86^{*}$ & $<0.01$ \\
\hline
\end{tabular}

Note: Comparison of treatment with control group $* \mathrm{P}<0.05$.

\section{Discussion}

RA is a disease mediated by chronic inflammation, which belongs to one of the immunological diseases, and is the most common clinically. Among them, the incidence of RA is mainly middle aged and aged, and the incidence is $0.3 \% \sim 0.4 \%$. Women are more likely to suffer from RA than men $^{[11,12]}$.

Joint pain, swelling, deformity, and loss of mobility in severe cases are the most obvious symptoms and signs of RA. Its complicated multi-system functional impairment, such as pulmonary interstitial lesions, osteoporosis, secondary Sjogren's syndrome, anemia and a series of changes, have brought some difficulties to the treatment and prognosis of $\mathrm{RA}^{[13]}$.

Although there are many drugs and methods for the treatment of RA at present, it is still mainly to control the disease. There is no specific drug for the cure of RA at present, so it is still necessary to further search for more effective treatment methods.

Zhuang doctors believe that feeling evil poison, weakness of vital qi, and imbalance of emotions are the main causes of RA, blocking the dragon and fire roads of joints and muscles is the key, and the virulent deficiency is the core ${ }^{[14]}$.

In terms of treatment, it is generally advocated that the main therapeutic principles should be holistic treatment, such as removing evil toxins, regulating Qi and blood, clearing collaterals and relieving pain, and unblocking roads [15].

External treatment in the treatment of choice, the zhuang medicine is relatively abundant, commonly used in zhuang medicine treatment has a strong $d$ thermal acupuncture, zhuang medicine fire needle therapy, zhuang medicine bamboo cans, zhuang medicine bath, etc., but in recent years, the zhuang medicine take orally in treating RA are obtained good treatment effect, such as zhuang medicine eight dragon diamond particles, zhuang medicine dragon drill through polioplus partners, zhuang medicine gaultheria yunnanensis in RA treatment also showed good therapeutic effect as ${ }^{[16]}$

Pangyuzhou professor as zhuang medicine academic leaders, to the zhuang medicine have deep understanding, is 
good at using zhuang medicine for clinical common disease, the treatment of RA has a wealth of experience, after continuous exploration and practice, summed up the new treatment method for the treatment of RA, the zhuang medicine comprehensive therapy, the therapy advocates both inside and outside simultaneously, supplemented by diet enhance curative effect.

The internal treatment is mainly through oral administration of the strong medicine Longzhuotong $\mathrm{Bi}$ Fang Granules, which are mainly composed of Feilongzhangxue, octagon maple, Dazhuana, Qingfengteng, Jiulong vine, Ziliangniacin and Five Fingermao peach root and other drugs, and have the effect of removing wind and dehumidifying, strengthening and tonifying deficiency, and clearing the stasis of the road.

External treatment is mainly the use of Zhuang medicine thermal sensitive acupuncture point treatment, the treatment at the same time includes Zhuang medicine thermal sensitive moxibustion and Zhuang medicine acupuncture therapy, with the effect of Bi pain relief, cold and dehumidification; Dietotherapy is mainly composed of black snake, ganoderma lucidum, coix seed and other drugs, to be taken inside the pigeon stew, has a good auxiliary treatment effect on rheumatoid.

Inside and outside governance combined therapy is not only promote one of the main therapy of modern medicine, and diet is generally become a kind of important auxiliary therapy, about diet, in huangdi neijing "when asked, dirty air method theory" with this kind of paper: "grain for raising, five fruit for help, five livestock for profit, five dishes for the filling, odor and clothing, to make up for the lean gas."

Through internal and external combination, the three-dimensional comprehensive therapy of Zhuang medicine dredges the network of three channels and two channels of the human body, regulates the synchronous movement of heaven, earth and human, and takes the medicine and food internally to nourish qi and strengthen the balance, so as to achieve the effect of both external and internal displacement, and promote the excretion of evil toxin from the body, thus playing a role in the treatment of RA.

This study is to observe the effect of Zhuang medicine three-dimensional comprehensive therapy on serum IL-6 expression level of patients with rheumatoid arthritis.

The results showed that the expression level of IL-6 could be decreased by the combination therapy of Zhuang medicine (P\&LT;0.05). It shows that the three-dimensional comprehensive therapy of Zhuang medicine has certain therapeutic significance for RA and is worthy of clinical promotion and application.

\section{Summary and prospect}

This preliminary study demonstrated the zhuang medicine comprehensive therapy for the treatment of RA with good effect, but there are still some limitations, this experiment is not yet clear zhuang medicine comprehensive therapy signaling pathways by which way or target genes involved in the regulation of IL - 6 , therefore, the future still need to further explore its research on signaling pathways or target genes, and it will be much help to clarify the mechanism of action of zhuang medicine comprehensive therapy.

In addition, more large, multicenter randomized controlled trials are needed to make the results more convincing.

\section{References}

[1] Nagai K, Matsubayashi K, Lde K, et al. Factors influencing placebo responses in rheumatoid arthritis clinical trials: a meta-analysis of randomized,double-blind, placebo-controlled studies [J]. Clin Drug Investig, 2020, 40(3): 197-209.

[2] Jeremy YN, Ashlee M Azizudin. Rheumatiod arthritis and osteoarthritis clinical practice guidelines provide few complementary and alternative medicine therapy recommendation:a systematie review $[\mathrm{J}]$. Clin Rheumatol, https://doi.org/10.1007/s10067-020-05054-y.

[3] Sarkar A, Sharma S, Agnihotri P, et al. Synovial fluid cell proteomic analysis identifies upregulation of alpha-taxilin proteins in rheumatiod arthritis:a potential prognostic marker $[\mathrm{J}] . \quad \mathrm{J}$ Immunol Res ,2020,2020:4897-983.

[4] Cai D, Hong S, Yang J, et al. The Effects of microRNA-515-5p on the Toll-Like Receptor 4 (TLR4)/JNK Signaling Pathway and WNT1-Inducible-Signaling Pathway Protein 1 (WISP-1) Expression in Rheumatoid Arthritis Fibroblast-Like Synovial (RAFLS) Cells Following Treatment with Receptor Activator of Nuclear Factor-kappa-B Ligand (RANKL) [J]. Med Sci Monit,2020,26:e920611.

[5] Zhi LQ, Zhong Q, Ma JB, et al. LncRNA H19 inhibitor 
represses synovial cell proliferation and apoptosis in rats with rheumatoid arthritis via Notch signaling pathway [J]. Europena Review Medical and Pharmacological Sciencens, 2020, 24: 4088-4094.

[6] Wang X, Chen J, Zhang R, et al. Interleukin-6 in Siberian sturgeon (acipenser baeri): molecular characterization and immune functional activity [J]. Fish \& Shellfish Immunology, 2020, 102.

[7] Fan XY, Hong MQ, Yang M. Research progress on the relationship between il- 6 and rheumatoid arthritis and mechanism of effect [J]. World Latest Medical Information Abstracts,2019,19(86): 65+69.

[8] Pang YZ, Lin C. Applied Zhuang Medicine Internal Medicine [M]. Nanning: Guangxi Science and Technology Press, 2002.

[9] He DC, Xiao JJ. Clinical efficacy and safety of bizhening in the treatment of rheumatoid arthritis [J]. Journal of Modern Integrated Traditional and Western Medicine, 2014, 23(28): 3090-3092+3095.

[10] Cai Y. Effects of leflunomide on TNF- $\alpha$, IL-6 and IL-17 in patients with rheumatoid arthritis [J]. Applied Medicine and Clinical Practice,2018,21(7): 802-806.

[11] Cao Y, Chen H, Hou HL, Yan JH. Effects of HebiFang combined with western medicine on serum and synovial fluid levels of IL-6, IL-8 and IL-17 in patients with rheumatoid arthritis $[\mathrm{J}]$. World Journal of Integrated Traditional Chinese and Western Medicine,
2020, 15(3): 497-500.

[12] Li T, Lv EJ. Effect of Guizhi Shaoxiaozhimu Decoction on serum levels of RF, IL-6 and GM-CSF in patients with rheumatoid arthritis $[\mathrm{J}]$. Harbin Pharmaceutical, 2018, 38(5): 476-478.

[13] Wang T, Li ZJ. Diagnosis and treatment of rheumatoid arthritis [J]. Chin J General Surg, 2020, 18(2): 170-171.

[14] Tang YZ, Ma FX, Chen YQ. Effect of Zhuang medicine Longzhuo Tongbi granule on Yin syndrome of rheumatoid arthritis [J]. Guangxi Medical Journal, 2020, 42(6): 769-772.

[15] Liao XT, Li FZ. Clinical research status of fire acupuncture therapy in the treatment of rheumatoid arthritis [J]. Hunan Journal of Traditional Chinese Medicine, 2020, 36(3):170-172.

[16] Li HJ. Study on quality control of strong medicine Baweilongzhuo granules and mechanism of anti-rheumatoid arthritis [D]. Chengdu University of Traditional Chinese Medicine, 2019.

[17] Pang YZ, Xing SS, Fang G, Pang XF. Clinical observation on the treatment of rheumatoid arthritis by Longzhuo Tongbi prescription [J]. Chinese Journal of Ethnic Medicine, 2013, 19(1): 1-2.

[18] Wang L, Lv JH, Liu Y, Rao WY, Deng YY, Qin L. Screening of active site and mechanism of Zhuang drug Touguxiang for anti-rheumatoid arthritis [J]. Pharmaceutical Research, 2019, 38(1): 8-11. 